\title{
Phase I study of dose escalation to dominant intraprostatic lesions using high-dose-rate brachytherapy
}

\author{
Christopher H. Chapman, MD, MS', Steve E. Braunstein, MD, PhD!, Jean Pouliot, PhD!, Susan M. Noworolski, PhD², \\ Vivian Weinberg, PhD', Adam Cunha, PhD', John Kurhanewicz, PhD², Alexander R. Gottschalk, MD, PhD?, \\ Mack Roach III, MD, FACR, FASTRO', I-Chow Hsu, MD, FACR, FASTRO' \\ 'Department of Radiation Oncology, ${ }^{2}$ Department of Radiology and Biomedical Imaging, University of California, San Francisco, \\ California, USA
}

\begin{abstract}
Purpose: Radiation dose escalation for prostate cancer improves biochemical control but is limited by toxicity. Magnetic resonance spectroscopic imaging (MRSI) can define dominant intraprostatic lesions (DIL). This phase I study evaluated dose escalation to MRSI-defined DIL using high-dose-rate (HDR) brachytherapy.

Material and methods: Enrollment was closed early due to low accrual. Ten patients with prostate cancer (T2a-3b, Gleason 6-9, PSA < 20) underwent pre-treatment MRSI, and eight patients had one to three DIL identified. The eight enrolled patients received external beam radiation therapy to $45 \mathrm{~Gy}$ and HDR brachytherapy boost to the prostate of 19 Gy in 2 fractions. MRSI images were registered to planning CT images and DIL dose-escalated up to $150 \%$ of prescription dose while maintaining normal tissue constraints. The primary endpoint was genitourinary (GU) toxicity.

Results: The median total DIL volume was $1.31 \mathrm{ml}$ (range, 0.67-6.33 ml). Median DIL boost was $130 \%$ of prescription dose (range, 110-150\%). Median urethra $\mathrm{V}_{120}$ was $0.15 \mathrm{ml}$ (range, 0-0.4 ml) and median rectum $\mathrm{V}_{75}$ was $0.74 \mathrm{ml}$ (range, 0.1-1.0 ml). Three patients had acute grade $2 \mathrm{GU}$ toxicity, and two patients had late grade 2 GU toxicity. No patients had grade 2 or higher gastrointestinal toxicity, and no grade 3 or higher toxicities were noted. There were no biochemical failures with median follow-up of 4.9 years (range, 2-8.5 years).

Conclusions: Dose escalation to MRSI-defined DIL is feasible. Toxicity was low but incompletely assessed due to limited patients' enrollment.

Key words: focal, image-guided brachytherapy, intraprostatic, prostate cancer.

\section{Purpose}

Multiple randomized trials have shown that escalating the dose of external beam radiation therapy (EBRT) beyond the historical standard of 65-70 Gy improves biochemical control of localized prostate cancer $[1,2,3,4,5,6]$. However, higher doses are limited by increased rates of gastrointestinal (GI) and genitourinary (GU) toxicity $[2,4,5]$. The intrinsic conformity of high-dose-rate (HDR) brachytherapy allows for dose escalation beyond what is safely achievable with external beam radiation techniques $[7,8]$. HDR brachytherapy has the additional advantage of hypofractionation, especially effective in prostate cancer due to its low alpha/beta ratio $[9,10]$. Accordingly, trials using brachytherapy boosts show improvement in biochemical control over dose-escalated external beam radiation alone $[11,12,13,14]$. However, further dose escalation to the entire prostate using brachytherapy has previously

been limited by normal tissue toxicity, particularly urinary toxicity associated with dose to the urethra $[15,16,17]$.

Due to the multifocal nature of prostate cancer, the conventional assumption in dose escalation trials has been that the entire gland must receive a high-dose. However, histopathologic studies have shown that a single or few dominant intraprostatic lesions (DIL) comprise the large majority of the tumor burden yet are typically $<10 \%$ of the total gland volume $[18,19]$. Magnetic resonance imaging (MRI) with spectroscopy (MRSI) allows for identification of DIL with high accuracy and reproducibility, providing a basis for image-guided therapy $[20,21,22]$. Dosimetric studies also have demonstrated the feasibility of focally increasing DIL dose to $120-150 \%$ of the whole prostate prescription dose while maintaining normal tissue dose constraints using inverse-planned HDR brachytherapy [23,24,25].
\end{abstract}

Address for correspondence: Christopher H. Chapman, MD, MS, Department of Radiation Oncology, University of California San Francisco, 1600 Divisadero St., Ste. H1031, San Francisco, CA 94143-1708, USA, phone: +1 415 353-7175, fax: +1 415 353-9883, 凶 e-mail: christopher.chapman@ucsf.edu
Received: 01.02 .2018

Accepted: 18.05 .2018

Published: 30.06 .2018 
With this background, the present study was opened in 2008 to further investigate dose escalation to MRSIdefined DIL using inverse-planned HDR brachytherapy, in addition to whole prostate brachytherapy and EBRT. We report results of this phase I trial.

\section{Material and methods}

Single-arm phase I trial enrolled patients with biopsyproven prostate adenocarcinoma. Planned total accrual was 25 patients. All patients were staged by the National Comprehensive Cancer Network (NCCN) guidelines and were free from nodal or distant metastatic disease. Eligible patients had intermediate- or high-risk localized prostate cancer within the following groups: clinical stage T2a-c, Gleason score 2-6, and prostate-specific antigen (PSA) 10-20 ng/ml; clinical stage T3a-b, Gleason score 2-6, and PSA $\leq 20$; or clinical stage T2a-3b, Gleason score 7-10, and PSA $\leq 20$. No prior radiation, prostate-ablative therapy, or androgen deprivation therapy (ADT) was permitted before study enrollment due to effects on prostate anatomy and metabolism [26]. Written informed consent was obtained from all subjects following a protocol approved by the institutional Committee of Human Research (10-04222) and the Department of Defense Human Research Protections Office (A-12494).

The primary outcome measure was the occurrence of GU toxicity scored using the National Cancer Institute Common Terminology Criteria for Adverse Events (CTCAE version 4) grading criteria. Preliminary experience indicated $<5 \%$ grade 3 or higher GU toxicity and approximately $15 \%$ grade 2 or higher GU toxicity occurring within 9 months after completing radiotherapy (acute GU toxicity) [27]. Therefore, for this trial if $>4$ of 25 patients (> 16\%) experienced grade 2 or higher GU toxicity within 9 months after completing radiotherapy, the treatment would be considered unacceptable and accrual discon- tinued. The upper bound of the exact $95 \%$ confidence interval for such toxicity is $33 \%$. In addition, if at any time $\geq 2$ patients experienced grade 3 or higher acute GU toxicity, accrual would be stopped.

After enrollment, patients underwent MRI of the prostate. Images were obtained using a 3T system with pelvic and endorectal radiofrequency coils, using previously described sequences to acquire T1-weighted, T2-weighted, and three-dimensional spectroscopic data on a $12 \times 8 \times 8$ grid with $5.4 \mathrm{~mm}$ cubic voxels [28]. Additional T2-weighted images were acquired without the endorectal coil, allowing for deformable registration to treatment planning images [28,29]. Spectroscopic voxels were scored using a standardized system from 1-5 with 5 representing the highest probability of malignancy [30]. A DIL was defined as contiguous voxels with a score of 4 or 5 .

After imaging, treatment began with ADT if prescribed by the treating physician, comprised of oral bicalutamide $50 \mathrm{mg}$ daily and intramuscular leuprolide $7.5 \mathrm{mg}$ monthly. All patients received EBRT to the whole prostate and seminal vesicles. Pelvic lymph nodes were also included in the treatment volume if the risk of nodal involvement was estimated at $>15 \%$ [31]. EBRT was delivered to a dose of $45 \mathrm{~Gy}$ in 25 daily fractions.

One to three weeks before or after EBRT, HDR temporary brachytherapy was performed using a 192-Ir source administered via transperineal catheters. All implants were comprised of 16 catheters inserted into the prostate using real-time transrectal ultrasound guidance as previously described [32]. For all patients, 16 catheters were used regardless of prostate size for consistency based on a prior modelling study [33]. After implantation, patients underwent pelvic computed tomography (CT) with $2-3 \mathrm{~mm}$ slice thickness for treatment planning and simulation. On the treatment planning $\mathrm{CT}$, the treating physician delineated the whole prostate clinical target volume (CTV) without margin and the organs at risk (OAR) includ-
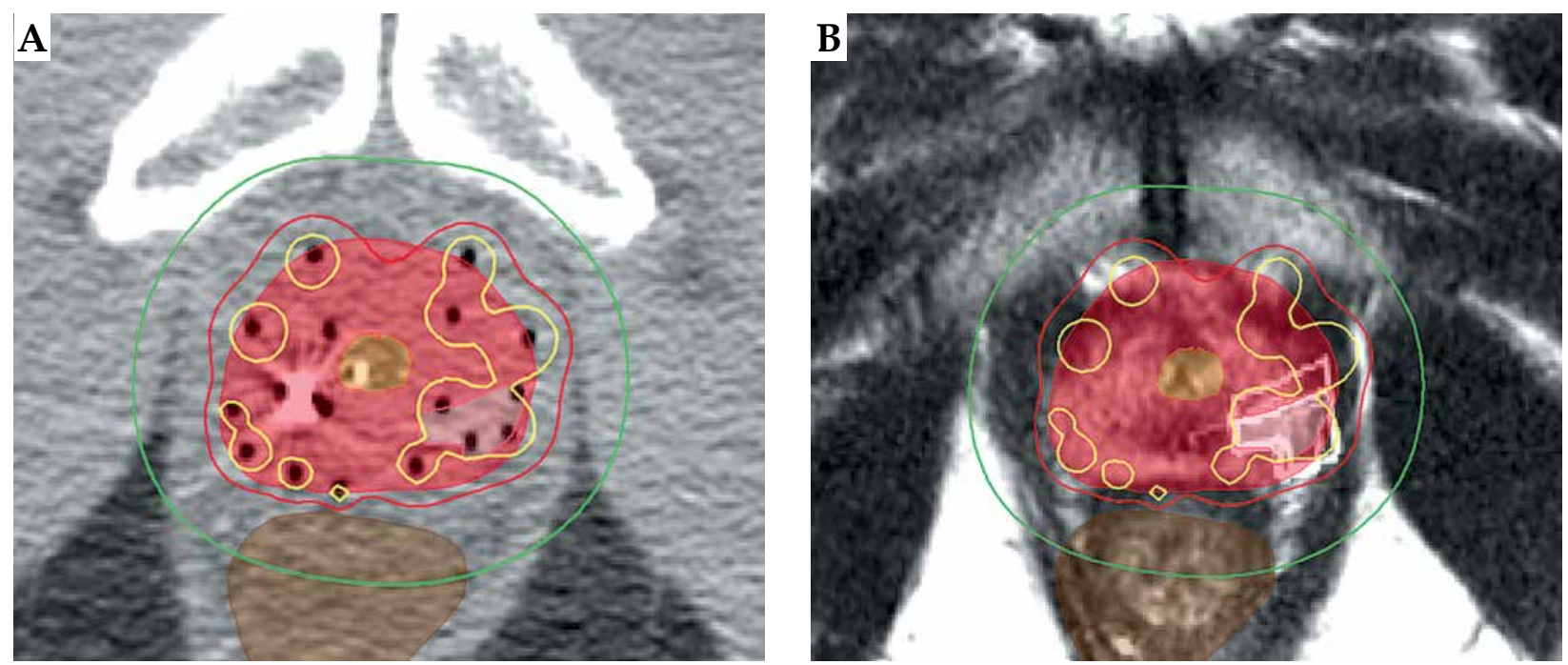

Fig. 1. Example treatment plan of patient treated with $150 \%$ boost of dominant intraprostatic lesions (DIL) in left peripheral zone. Shaded areas are prostate clinical target volume (CTV), urethra, DIL, and rectum. Isodose lines are 50\%, 100\%, and 150\% of prescription dose. A) treatment planning computed tomography (CT). B) T2-weighted magnetic resonance imaging with DIL outline registered to treatment planning CT 
ing the intraprostatic urethra, periprostatic rectum, and periprostatic bladder. The DIL volume as defined on the pre-treatment endorectal coil MRSI was transformed by non-linear local weighted-mean registration with mutual information to the pre-treatment MRI without endorectal coil, as previously reported [28]. This was then transformed by rigid body registration to the treatment planning CT (Figure 1). Both transformation steps have registration errors of $<2 \mathrm{~mm}[28,29]$. No planning margin was used for the focal DIL dose-escalation, as the entire prostate was receiving a therapeutic dose. Treatment planning was performed using inverse planning with simulated annealing (IPSA; Oncentra Brachy, Elekta, Sweden) [34]. Treatment planning goals were defined by dose-volumes normalized to the prescription dose, where $\mathrm{D}_{\mathrm{x}}$ is the minimum dose to $\mathrm{x} \%$ of the target volume, or where $V_{x}$ is the volume encompassing $x \%$ of the prescription dose. Target goals were whole prostate CTV $\mathrm{D}_{90} \geq 100 \%$ prescription dose and a simultaneous boost to the DIL $\mathrm{D}_{90}$ up to $150 \%$ while maintaining OAR dose-volume constraints [23]. OAR constraints were as follows: urethra $\mathrm{V}_{120} \leq 1 \mathrm{ml}$ and $\mathrm{V}_{150}=0 \mathrm{ml}$; rectum $\mathrm{V}_{75} \leq 1 \mathrm{ml}$; bladder $V_{75} \leq 1 \mathrm{ml}$. All patients received a whole prostate CTV prescription dose of $9.5 \mathrm{~Gy} \times 2$ fractions (19 Gy total) at least 6 hours apart using a single implant session.

For this study, acute and late genitourinary (GU) and gastrointestinal (GI) toxicity were graded by the CTCAE version 4. Patient-reported outcomes included GU and GI symptoms measured using the International Prostate Symptom Score (IPSS) and the Sexual Health Inventory for Men (SHIM), which were obtained pre-treatment, one month, 3 months, 6 months, 9 months, and 12 months post-treatment $[35,36]$. Patient reported outcomes included subscales from the Extended Prostate Cancer Index Composite for Clinical Practice (EPIC-CP) for overall urinary function, bowel function, and sexual function, collected at 6 months and 12 months post-treatment [37]. Disease outcomes included PSA nadir (lowest observed PSA after completion of radiation and hormonal therapy before any PSA rise), freedom from biochemical failure (FFBF) by the ASTRO Phoenix definition (nadir $+2 \mathrm{ng} / \mathrm{ml}$ ), cause-specific death, and death due to any cause. Patient records were cross-referenced to the institutional cancer registry to obtain information from other institutions.

\section{Results}

Trial enrollment was open from June 2008 to January 2011. Seventeen patients were assessed for eligibility, and 10 underwent pre-treatment prostate MRSI. Of the 7 patients who were excluded, 5 did not satisfy the eligibility criteria and 2 declined enrollment. Of the remaining 10 patients who underwent imaging, one patient was excluded due to no DIL identified on MRSI, and one patient was excluded due to technical imaging error, leaving eight patients eligible for DIL dose escalation per study protocol (Figure 2). Clinical characteristics are listed in Table 1. The median age was 61, and median pre-treatment PSA was $9.9 \mathrm{ng} / \mathrm{ml}$ (range, $1.0-19.5 \mathrm{ng} / \mathrm{ml}$ ). Five patients were NCCN unfavorable intermediate-risk, two were standard high-risk, and one was very high-risk (T3b and Gleason score 5+4). All patients received EBRT per protocol with

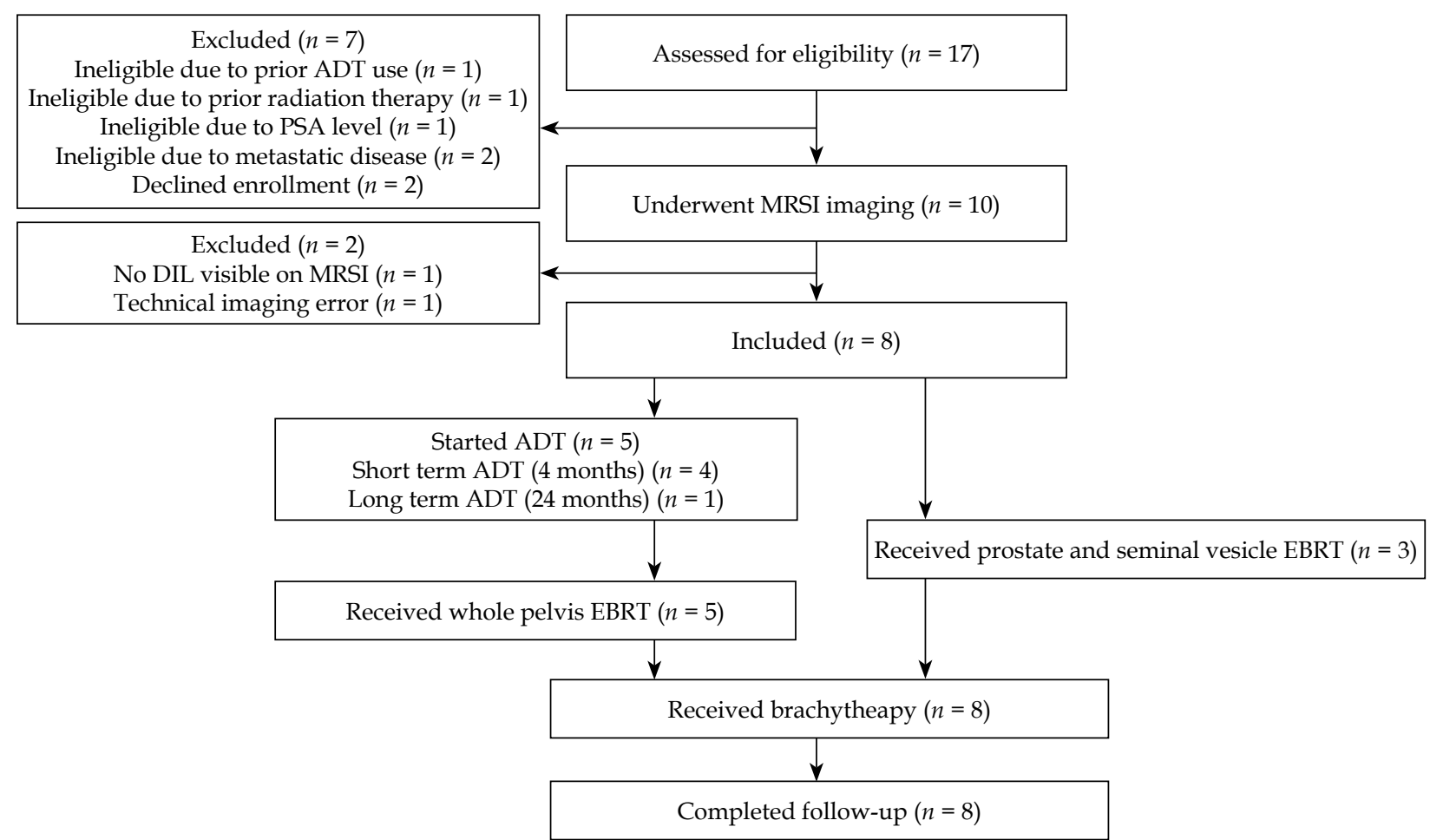

ADT - androgen deprivation therapy, PSA - prostate-specific antigen, MRSI - magnetic resonance imaging with spectroscopy, DIL - dominant intraprostatic lesions, EBRT - external beam radiation therapy

Fig. 2. Eligibility and inclusion flowchart 


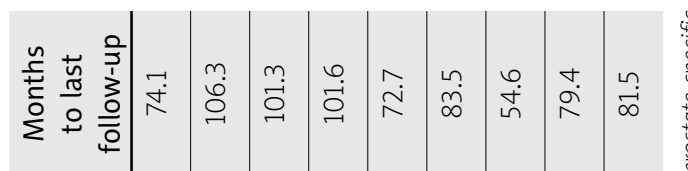

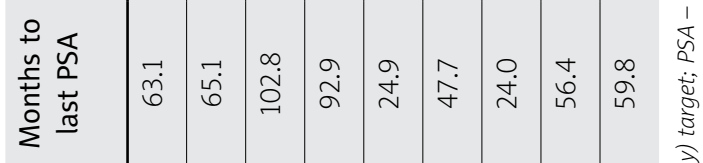

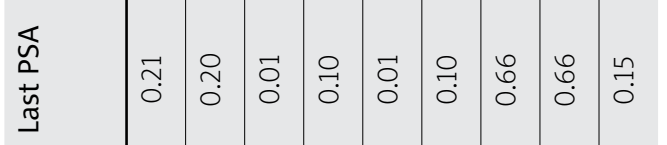

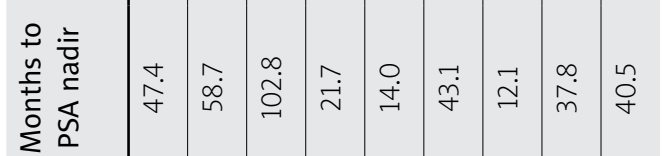

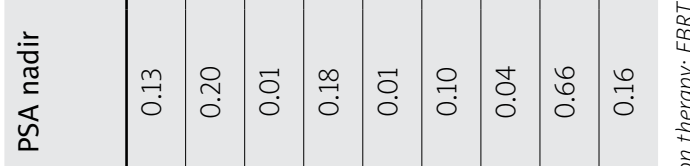

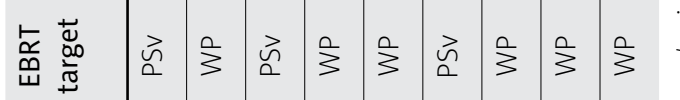

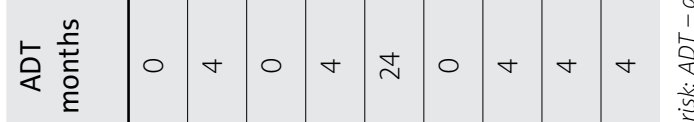

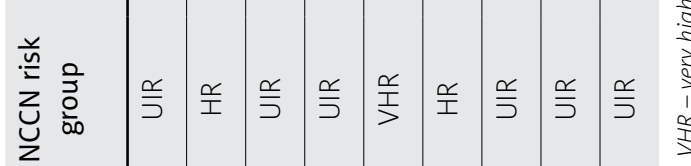

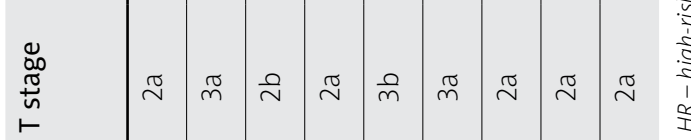

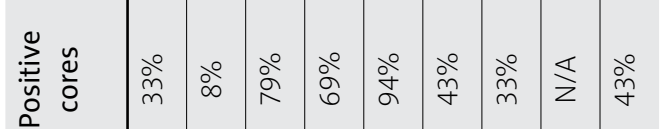

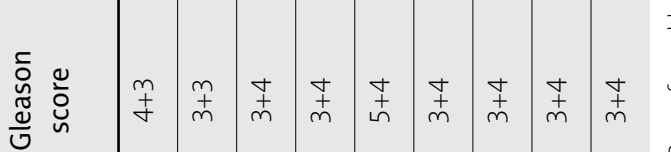

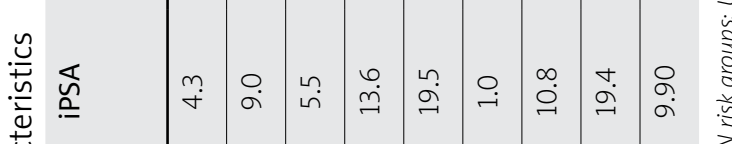

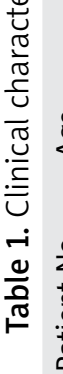

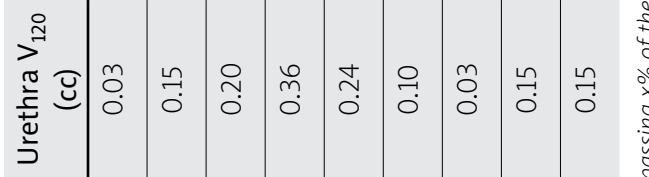

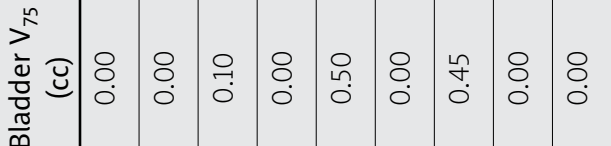

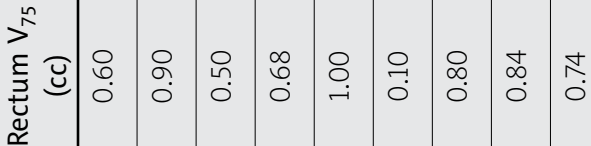

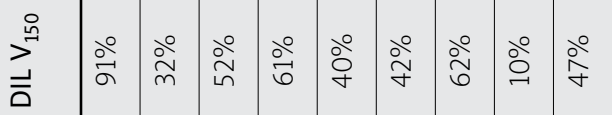

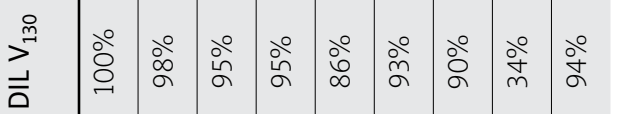

吾

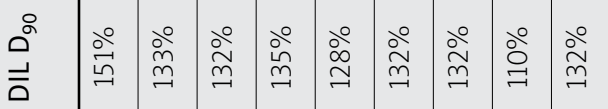

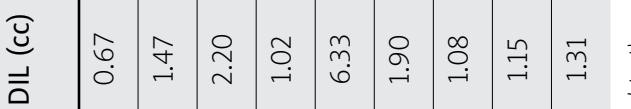

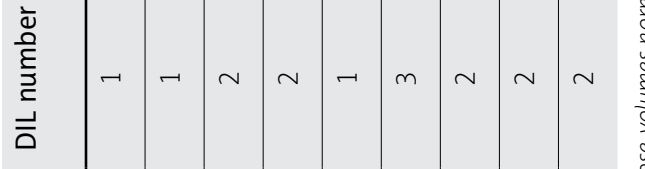

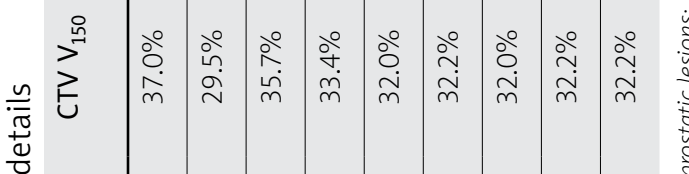

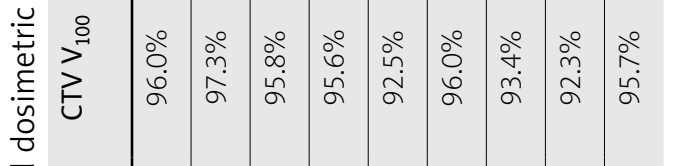

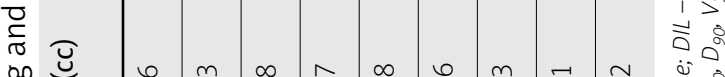

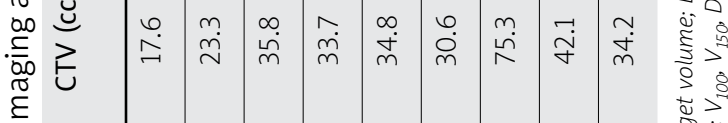

离 
$45 \mathrm{~Gy}$ in 25 fractions to the prostate and seminal vesicles. In five patients, the treatment fields also included the pelvic lymph nodes; they received ADT starting before EBRT. Four patients received ADT for 4 months duration (three intermediate- and one high-risk patient), and one for 24 months duration (very high-risk patient).

All patients received HDR brachytherapy per protocol. Imaging and dosimetric details are listed in Table 2. The median prostate volume by MRI was $34.3 \mathrm{ml}$ (range, 17.6-75.3 ml). Three patients had a single DIL, four patients had two DILs, and one patient had three DILs. The median total DIL volume was $1.31 \mathrm{ml}$ (range, 0.67$6.33 \mathrm{ml}$ ), which was a median of $5.0 \%$ of the total prostate volume (range, 1.4-18.2\%). The median prostate $V_{100}$ was 95.7\% (range, 92.3-97.3\%), and median $\mathrm{V}_{150}$ was $32.2 \%$ (range, 29.5-37.0\%). Five patients received DIL boost to $130 \%$, one patient to $110 \%$, one to $125 \%$, and one to $150 \%$ (defined as $\geq 90 \%$ coverage). The actual median $\mathrm{D}_{90}$ across patients was $132 \%$ of prescription dose (range, 110 $151 \%)$. All OAR constraints were achieved in all patients. The median duration of the entire radiation treatment course was 7.4 weeks (range, 6.1-9.1 weeks). The median time from MRI to brachytherapy was 10.9 weeks (range, 6.6-23.7 weeks).

Toxicity results are represented in Figure 3 . Three patients had acute grade 2 GU toxicity consisting of nocturia, urgency, and obstructive symptoms. Four patients had acute grade $1 \mathrm{GU}$ toxicity of increased urinary frequency. Two patients had late grade 2 GU toxicity at 1-year post-treatment: 1 patient with a urethral stricture requiring urethral dilation, and 1 patient with dysuria/ hematuria requiring oral anti-inflammatory medications. One of the patients with late grade 2 GU toxicity also had acute grade $1 \mathrm{GU}$ toxicity, and the other had no acute GU toxicity. No patients had any acute or late GU toxicity of grade 3 or higher. For GI toxicity, 4 patients had acute grade $1 \mathrm{GI}$ toxicity of diarrhea. One patient with acute GI toxicity also had late grade 1 GI toxicity of diarrhea persisting beyond 9 months post-treatment. No patient had any acute or late GI toxicity of grade 2 or higher.

Median scores and changes from pre-treatment for IPSS and SHIM are reported in Table 3. On the IPSS

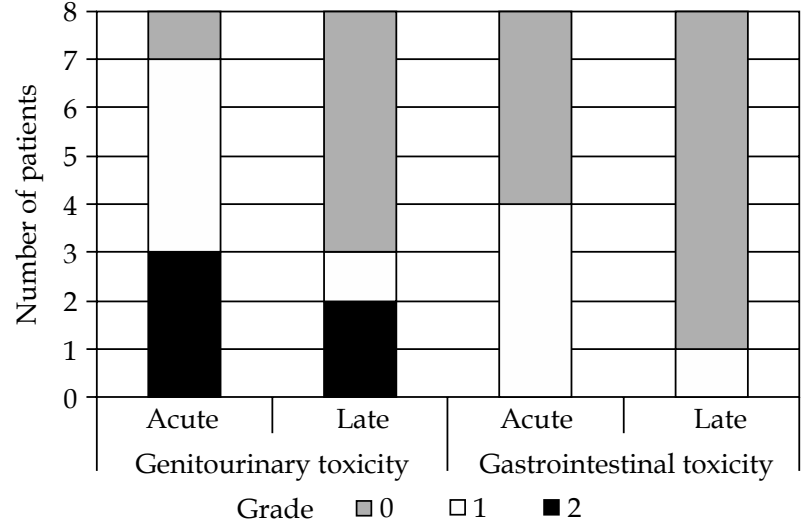

Fig. 3. Treatment toxicity by grade, CTCAE version 4

questionnaire for urinary symptoms (possible 0-35 with higher scores representing more symptoms), the median pre-treatment score was 7.5 (range, 3-13). IPSS was increased from pre-treatment by a median of 6.5 at 1 -month post-treatment and by a median of 1 at 3 months post treatment, but by 6, 9, and 12 months post-treatment, the median change in IPSS was the same or better than pre-treatment IPSS (Figure 4). On the SHIM scale for sexual function (possible 1-25 with higher scores representing better function), the median pre-treatment score was 18.5 (range, 1-25). SHIM scores decreased by a median of 3 at 1-month post-treatment and by a median of 8 at 3 months post-treatment. The median change in SHIM was negative for all post-treatment time points. At12months post-treatment, the three patients whodid not receive ADT had SHIM scores of 6 (-8 from pre-treatment), 8 (-9 from pre-treatment), and 25 (same as pre-treatment).

Subscales from the EPIC-CP prostate symptoms quality of life questionnaire were administered at 6 and 12 months after radiation therapy [37]. Patients were asked how much of a problem overall urinary, bowel, and sexual function had been for them over the last 4 weeks, using a 5-level scale ranging from 0 (no problem) to 4 (big problem). Of the 8 enrolled patients, 6 completed the questionnaire at 6 months after radiation, and 4 completed the questionnaire at 12 months after radiation. Results

Table 3. Median scores and changes from pre-treatment for the International Prostate Symptom Score (IPSS) and the Sexual Health Inventory for Men (SHIM)

\begin{tabular}{lcccccc} 
& Pre-treat & 1 month & 3 months & 6 months & 9 months & 12 months \\
\hline IPSS & 8 & 8 & 8 & 7 & 7 & 8 \\
\hline \begin{tabular}{lcccc} 
Score \\
\hline Change
\end{tabular} & $7.5(3,13)$ & $15.5(5,20)$ & $10(2,19)$ & $7(2,22)$ & $6(2,23)$ & $7.5(2,16)$ \\
\hline SHIM & N/A & $+6.5(-6,+15)$ & $+1(-10,+9)$ & $+0(-7,+12)$ & $-2(-6,+10)$ & $-2(-4,+9)$ \\
\hline$n$ & 8 & & & & 6 & 7 \\
\hline Score & $18.5(1,25)$ & $18(1,25)$ & $7.5(2,25)$ & $5.5(1,23)$ & $13(1,25)$ & $8(1,25)$ \\
\hline Change & N/A & $-3(-23,+4)$ & $-8(-17,+2)$ & $-3(-16,+1)$ & $-0.5(-13,+1)$ & $-6(-23,+1)$
\end{tabular}

Values are written as median (minimum, maximum); IPSS - International Prostate Symptom Score; SHI - the Sexual Health Inventory for Men 


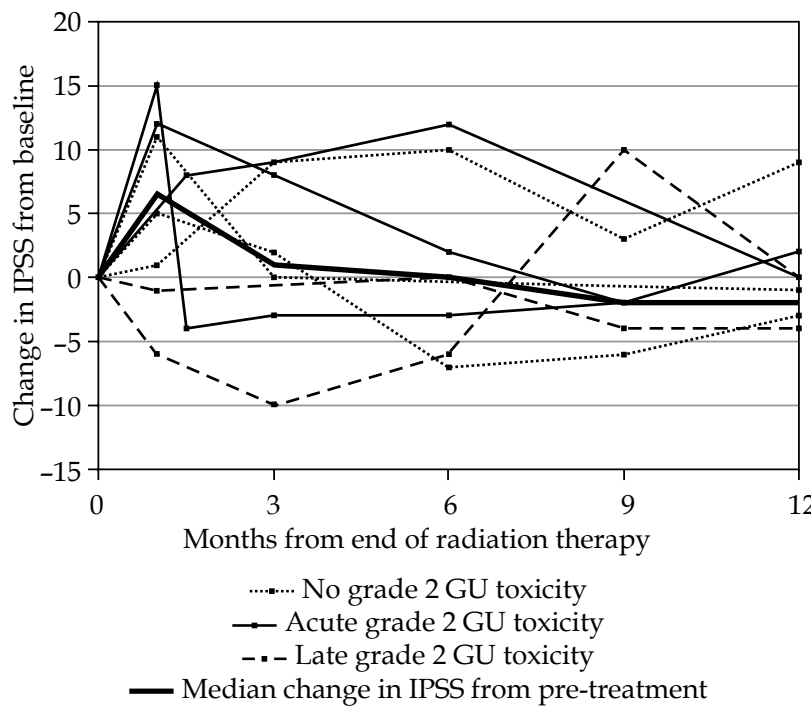

Fig. 4. Change in IPSS from pre-treatment score by individual patient and median change up to 12 months posttreatment

are presented in Table 4. For urinary and bowel function, no patient responded higher than 2 (small problem) at any time point, and 3 patients responded 0 (no problem) at both 6 and 12 months. For sexual function, 1 patient responded 4 (big problem) at 6 months and 2 responded 4 (big problem) at 12 months, but the remainder responded 0-2.

The median PSA nadir was $0.12 \mathrm{ng} / \mathrm{ml}$ (range, 0.01$0.66 \mathrm{ng} / \mathrm{ml}$ ). The median time to nadir after completing all radiation therapy was 40.5 months (range, 12.1-102.8 months). There was one outlier who reached a relative nadir of $0.21 \mathrm{ng} / \mathrm{ml}$ after 14.7 months before rising again, but then achieved a PSA of 0.01 with later follow-up at 102.8 months. Other than this, the longest time to PSA nadir was 58.7 months. For the three patients not receiving ADT, PSA nadirs were $0.13,0.21$, and $0.10 \mathrm{ng} / \mathrm{ml}$, decreased from pre-treatment PSAs of 4.29, 5.50, and $1.00 \mathrm{ng} / \mathrm{ml}$, respectively. The times to PSA nadir for these patients not receiving ADT were 47.4 months, 14.7 months, and 43.1 months, respectively. After a median of 4.9 years of available PSA follow-up (range, 2-8.5 years), there were no biochemical failures. Survival data were available with a median follow-up of 6.7 years (range, 4.5-8.7 years). As of last follow-up, there were no deaths from any cause.

\section{Discussion}

This trial demonstrates clinical feasibility of dose escalation to MRSI-defined DIL using inverse-planned HDR brachytherapy. The level of dose escalation was tailored to maintain OAR dose constraints, and the majority of patients achieved DIL boosts $\geq 130 \%$ of prescription dose without any OAR dose constraint violations. The primary adverse effect was urinary toxicity, with 3 patients experiencing acute grade $2 \mathrm{GU}$ toxicity. No grade 3 or higher toxicities by CTCAE criteria were noted. Two patients experienced late urinary toxicities requiring interventions; however, these resolved without permanent organ dysfunction. Acute urinary symptoms were generally temporary, as demonstrated by IPSS returning to near pre-treatment baseline or better by 6 months post-treatment for most patients, similar to other studies using EBRT plus HDR brachytherapy $[38,39,40]$. By 12 months post-treatment, all patients except one had IPSS within 4 points of their pre-treatment score, which is within the test-retest variability for IPSS [41]. While the study was ended early due to poor accrual, the pre-defined toxicity threshold to end accrual of $>4$ patients having grade 2 or higher toxicity by 9 months post-treatment was not met. However, the study was very close to reach the pre-defined safety endpoint, as only 1 of the remaining 17 planned patients could have had grade 2 or higher GU toxicity to remain below the safety threshold. Thus, we consider the study inconclusive for the primary endpoint of toxicity.

Table 4. Response to overall function subscales of EPIC-CP at 6 and 12 months after radiation therapy. Patients were asked how much of a problem urinary, bowel, and sexual function had been over the last 4 weeks. $0=$ no problem, 1 = very small problem, $2=$ small problem, $3=$ moderate problem, 4 = big problem. NR - no response

\begin{tabular}{|c|c|c|c|c|c|c|}
\hline \multirow[t]{2}{*}{ Patient } & \multicolumn{3}{|c|}{6 months } & \multicolumn{3}{|c|}{12 months } \\
\hline & Urinary & Bowel & Sexual & Urinary & Bowel & Sexual \\
\hline 1 & $N R$ & NR & $N R$ & NR & NR & NR \\
\hline 2 & 0 & 2 & 1 & NR & NR & NR \\
\hline 3 & 0 & 0 & 4 & 0 & 0 & 4 \\
\hline 4 & 1 & 1 & 2 & NR & NR & NR \\
\hline 5 & 0 & 0 & 0 & 0 & 0 & 4 \\
\hline 6 & NR & NR & $N R$ & NR & NR & NR \\
\hline 7 & 1 & 2 & 0 & 2 & 1 & 0 \\
\hline 8 & 0 & 0 & 1 & 0 & 0 & 2 \\
\hline Median & 0 & 0.5 & 1 & 0 & 0 & 3 \\
\hline
\end{tabular}


Looking at the dosimetry results alone, GU toxicity rates would be expected to be acceptable. In the present study, the median urethra $\mathrm{V}_{120}$ was $0.15 \mathrm{ml}$ and maximum $0.36 \mathrm{ml}$ (Table 2). RTOG 0321 reported a mean urethral $\mathrm{V}_{125}$ of $0.15 \mathrm{ml}$ and maximum $0.94 \mathrm{ml}$, and an acute GU toxicity grade $\geq 2$ rate of $10 \%$ [27]. Urethral dose was also found to be the most predictive of GU toxicity in that trial [16]. Although the size and duration of the study are insufficient to evaluate disease outcomes, the results were encouraging with no biochemical failures after a median PSA follow-up of 4.9 years.

HDR brachytherapy has the relative advantage of adaptive treatment planning. Our experience in the present study and others indicate that a free-hand inverse planning technique allows for sufficient dose modulation to accommodate DIL boost without violating OAR constraints [42]. HDR brachytherapy has been previously used for intraprostatic DIL boosts with low toxicity reported. Borghede et al. treated 50 patients with 50 Gy EBRT followed by two fractions of 10 Gy using HDR brachytherapy, with dose escalation to 15 Gy per fraction in biopsy-verified tumor areas in 30 patients [43]. Disease-free survival was $96 \%$ at a mean follow-up of 45 months, with $8 \%$ grade 3 urinary toxicity and no highgrade GI toxicity by RTOG criteria. Two other more recent studies have reported the use of HDR brachytherapy to dose-escalate MRI-defined DIL. Gomez-Iturriaga et al. treated 15 patients with hypofractionated EBRT (37.5 Gy in 15 fractions), followed by a 15 Gy single fraction HDR brachytherapy boost [40]. Using inverse-planning, they achieved 131-151\% DIL dose escalation while maintaining a conservative urethral maximum dose of $115 \%$, and rectal $\mathrm{V}_{70}<1 \mathrm{ml}$. DIL volumes were similar to the present study, suggesting DIL dose escalation with more conservative OAR constraints is still possible. They reported $27 \%$ grade 2 GU toxicity and no grade 3 toxicity by CTCAE criteria. Vigneault et al. treated 19 patients with intermediate-risk prostate cancer with $45 \mathrm{~Gy}$ in 25 fractions EBRT followed by a 15 Gy single fraction HDR brachytherapy boost [39]. A single MRI-defined DIL per patient was dose-escalated to only $120 \%$, although the DIL tended to be larger than in the present study. In that study, $16 \%$ of patients had acute grade $2 \mathrm{GU}$ toxicity and one patient had acute grade 3 GU toxicity by RTOG criteria. There was no late grade 3 or higher toxicity. Early disease outcomes were promising with $95 \%$ biochemical control at 5 years.

The greatest limitation of the present study is the small size, with only 8 protocol eligible patients. The primary reasons for low enrollment were concurrent competing trials and the specification of no ADT before MRI spectroscopy, as many patients were referred having already started ADT. This restriction was enforced due to the known effects of ADT on MRSI sensitivity [26]. Since the design of this trial, there has been increased standardization of prostate multi-parametric MRI (mp-MRI) incorporating diffusion weighted imaging, dynamic contrast enhanced imaging as well as MRSI, resulting in the PI-RADS system [44]. Including other MRI modalities, it allows for DIL identification even within 3-4 months of starting ADT $[45,46]$, which should be considered in design of future trials.
Another key limitation of the present study was the potential for inaccurate localization of the DIL on the treatment planning CT. One component of this error is the uncertainty of image registration between MRSI (with endorectal coil) and CT (no endorectal coil). Using non-linear registration to an intermediate $\mathrm{MR}$ image without endorectal coil before registering to $\mathrm{CT}$, our image registration error is estimated at $<2 \mathrm{~mm}[28,29]$. Another component of the error is anatomic changes to the prostate due to ADT during the period between MRSI and treatment planning (median 10.9 weeks in this study). ADT is effective at decreasing prostate volume and has been used as a treatment for benign prostatic hypertrophy [47]. Changes in prostate size between MRSI and planning CT could create registration errors that would not be corrected for by rigid-body registration, and this was not examined in our study. One possible solution to this component of error would be obtaining the MRSI at the time of treatment, potentially as part of MRI-based treatment planning [48]. Despite the potential for inaccurate DIL localization in this study, no planning margin around the DIL was used. This was considered acceptable because the remainder of the prostate was still receiving a therapeutic dose, and is consistent with other studies using HDR brachytherapy for DIL dose-escalation [39,40,49].

\section{Conclusions}

Focal dose escalation to MRSI-defined DIL is feasible using inverse-planned HDR brachytherapy. Treatment toxicity met pre-defined thresholds, although the study was inconclusive due to low accrual. Patient-reported urinary symptoms attributable to treatment were generally near baseline by 6 months after treatment. Biochemical disease control was $100 \%$ at a median 4.9 years follow-up, but larger studies with longer follow-up are needed.

\section{Acknowledgements}

This work was supported by the US Department of Defense Prostate Cancer Research Program grant DOD-PC 030909.

\section{Disclosure}

The authors report no conflict of interest.

\section{References}

1. Eade TN, Hanlon AL, Horwitz EM et al. What dose of externalbeam radiation is high enough for prostate cancer? Int J Radiat Oncol Biol Phys 2007; 68: 682-689.

2. Kuban DA, Tucker SL, Dong L et al. Long-term results of the M.D. Anderson randomized dose-escalation trial for prostate cancer. Int J Radiat Oncol Biol Phys 2008; 70: 67-74.

3. Zelefsky MJ, Yamada Y, Fuks Z et al. Long-term results of conformal radiotherapy for prostate cancer: impact of dose escalation on biochemical tumor control and distant metastases-free survival outcomes. Int J Radiat Oncol Biol Phys 2008; 71: 1028-1033.

4. Al-Mamgani A, van Putten WLJ, Heemsbergen WD et al. Update of Dutch multicenter dose-escalation trial of radiotherapy for localized prostate cancer. Int J Radiat Oncol Biol Phys 2008; 72: 980-988. 
5. Beckendorf V, Guerif S, Le Prisé E et al. 70 Gy versus 80 Gy in localized prostate cancer: 5-year results of GETUG 06 randomized trial. Int J Radiat Oncol Biol Phys 2011; 80: 1056-1063.

6. Dearnaley DP, Jovic G, Syndikus I et al. Escalated-dose versus control-dose conformal radiotherapy for prostate cancer: long-term results from the MRC RT01 randomised controlled trial. Lancet Oncol 2014; 15: 464-473.

7. Hsu IC, Pickett B, Shinohara K et al. Normal tissue dosimetric comparison between HDR prostate implant boost and conformal external beam radiotherapy boost: potential for dose escalation. Int J Radiat Oncol Biol Phys 2000; 46: 851-858.

8. Georg D, Hopfgartner J, Gòra J et al. Dosimetric considerations to determine the optimal technique for localized prostate cancer among external photon, proton, or carbon-ion therapy and high-dose-rate or low-dose-rate brachytherapy. Int J Radiat Oncol Biol Phys 2014; 88: 715-722.

9. Brenner DJ, Hall EJ. Fractionation and protraction for radiotherapy of prostate carcinoma. Int J Radiat Oncol Biol Phys 1999; 43: 1095-1101.

10. Fowler JF. The radiobiology of prostate cancer including new aspects of fractionated radiotherapy. Acta Oncologica 2009; 44: 265-276.

11. Deutsch I, Zelefsky MJ, Zhang Z et al. Comparison of PSA relapse-free survival in patients treated with ultra-high-dose IMRT versus combination HDR brachytherapy and IMRT. Brachytherapy 2010; 9: 313-318.

12. Hoskin PJ, Rojas AM, Bownes PJ et al. Randomised trial of external beam radiotherapy alone or combined with highdose-rate brachytherapy boost for localised prostate cancer. Radiother Oncol 2012; 103: 217-222.

13. Spratt DE, Zumsteg ZS, Ghadjar P et al. Comparison of highdose (86.4 Gy) IMRT vs combined brachytherapy plus IMRT for intermediate-risk prostate cancer. Br J Urol Int 2014; 114: 360-367.

14. Morris WJ, Tyldesley S, Rodda S et al. Androgen suppression combined with elective nodal and dose escalated radiation therapy (the ASCENDE-RT Trial): An analysis of survival endpoints for a randomized trial comparing a low-dose-rate brachytherapy boost to a dose-escalated external beam boost for high- and intermediate-risk prostate cancer. Int J Radiat Oncol Biol Phys 2017; 98: 275-285.

15. Rodda S, Tyldesley S, Morris WJ et al. ASCENDE-RT: An analysis of treatment-related morbidity for a randomized trial comparing a low-dose-rate brachytherapy boost with a dose-escalated external beam boost for high- and intermediate-risk prostate cancer. Int J Radiat Oncol Biol Phys 2017; 98: 286-295.

16. Hsu I-C, Hunt D, Straube W et al. Dosimetric analysis of radiation therapy oncology group 0321: the importance of urethral dose. Pract Radiat Oncol 2014; 4: 27-34.

17. Raleigh DR, Chang AJ, Tomlin B et al. Patient- and treatment-specific predictors of genitourinary function after high-dose-rate monotherapy for favorable prostate cancer. Brachytherapy 2015; 14: 795-800.

18. Wise AM, Stamey TA, McNeal JE et al. Morphologic and clinical significance of multifocal prostate cancers in radical prostatectomy specimens. Urology 2002; 60: 264-269.

19. Hollmann BG, van Triest B, Ghobadi G et al. Gross tumor volume and clinical target volume in prostate cancer: How do satellites relate to the index lesion. Radiother Oncol 2015; 115: 96-100.

20. Scheidler J, Hricak H, Vigneron DB et al. Prostate cancer: localization with three-dimensional proton MR spectroscopic imaging - clinicopathologic study. Radiology 1999; 213: 473-480.

21. Brame RS, Zaider M, Zakian KL et al. Regarding the focal treatment of prostate cancer: inference of the Gleason grade from magnetic resonance spectroscopic imaging. Int J Radiat Oncol Biol Phys 2009; 74: 110-114.
22. Pickett B, Vigneault E, Kurhanewicz J et al. Static field intensity modulation to treat a dominant intra-prostatic lesion to 90 Gy compared to seven field 3-dimensional radiotherapy. Int J Radiat Oncol Biol Phys 1999; 44: 921-929.

23. Pouliot J, Kim Y, Lessard E et al. Inverse planning for HDR prostate brachytherapy used to boost dominant intraprostatic lesions defined by magnetic resonance spectroscopy imaging. Int J Radiat Oncol Biol Phys 2004; 59: 1196-1207.

24. Kim Y, Hsu I-C, Lessard E et al. Class solution in inverse planned HDR prostate brachytherapy for dose escalation of DIL defined by combined MRI/MRSI. Radiother Oncol 2008; 88: 148-155.

25. Cunha JAM, Pouliot J, Weinberg V et al. Urethra low-dose tunnels: validation of and class solution for generating urethra-sparing dose plans using inverse planning simulated annealing for prostate high-dose-rate brachytherapy. Brachytherapy 2012; 11: 348-353.

26. Mueller-Lisse UG, Swanson MG, Vigneron DB et al. Time-dependent effects of hormone-deprivation therapy on prostate metabolism as detected by combined magnetic resonance imaging and 3D magnetic resonance spectroscopic imaging. Magn Reson Med 2001; 46: 49-57.

27. Hsu IC, Bae K, Shinohara $\mathrm{K}$ et al. Phase II trial of combined high-dose-rate brachytherapy and external beam radiotherapy for adenocarcinoma of the prostate: preliminary results of RTOG 0321. Int J Radiat Oncol Biol Phys 2010; 78: 751-758.

28. Reed G, Cunha JA, Noworolski S et al. Interactive, multi-modality image registrations for combined MRI/MRSI-planned HDR prostate brachytherapy. J Contemp Brachytherapy 2011; 3: 26-31.

29. Alterovitz R, Goldberg K, Pouliot J et al. Registration of MR prostate images with biomechanical modeling and nonlinear parameter estimation. Med Phys 2006; 33: 446-454.

30. Jung JA, Coakley FV, Vigneron DB et al. Prostate depiction at endorectal MR spectroscopic imaging: investigation of a standardized evaluation system. Radiology 2004; 233: 701-708.

31. Roach M 3rd, Marquez C, Yuo HS et al. Predicting the risk of lymph node involvement using the pre-treatment prostate specific antigen and Gleason score in men with clinically localized prostate cancer. Int J Radiat Oncol Biol Phys 1994; 28: 33-37.

32. Kim Y, Hsu IC, Pouliot J. Measurement of craniocaudal catheter displacement between fractions in computed tomography-based high dose rate brachytherapy of prostate cancer. J Appl Clin Med Phys 2007; 8: 2415-2427.

33. Charra-Brunaud C, Hsu I-CJ, Weinberg V et al. Analysis of interaction between number of implant catheters and dose-volume histograms in prostate high-dose-rate brachytherapy using a computer model. Int J Rad Oncol Biol Phys 2003; 56: 586-591.

34. Lachance B, Béliveau-Nadeau D, Lessard E et al. Early clinical experience with anatomy-based inverse planning dose optimization for high-dose-rate boost of the prostate. Int J Radiat Oncol Biol Phys 2002; 54: 86-100.

35. Barry MJ, Fowler FJ, O'Leary MP et al. The American Urological Association symptom index for benign prostatic hyperplasia. J Urol 1992; 148: 1549-1557.

36. Vroege JA. The sexual health inventory for men (IIEF-5). Int J Impot Res 1999; 11: 177.

37. Chang P, Szymanski KM, Dunn RL et al. Expanded Prostate Cancer Index Composite for Clinical Practice: development and validation of a practical health related quality of life instrument for use in the routine clinical care of patients with prostate cancer. J Urol 2011; 186: 865-872.

38. Morton GC, Loblaw DA, Sankreacha R et al. Single-fraction high-dose-rate brachytherapy and hypofractionated external beam radiotherapy for men with intermediate-risk prostate 
cancer: analysis of short- and medium-term toxicity and quality of life. Int J Radiat Oncol Biol Phys 2010; 77: 811-817.

39. Vigneault E, Mbodji K, Racine L-G et al. Image-guided highdose-rate brachytherapy boost to the dominant intraprostatic lesion using multiparametric magnetic resonance imaging including spectroscopy: Results of a prospective study. Brachytherapy 2016; 15: 746-751.

40. Gomez-Iturriaga A, Casquero F, Urresola A et al. Dose escalation to dominant intraprostatic lesions with MRI-transrectal ultrasound fusion High-Dose-Rate prostate brachytherapy. Prospective phase II trial. Radiother Oncol 2016; 119: 91-96.

41. El Din KE, Koch WF, de Wildt MJ et al. Reliability of the International Prostate Symptom Score in the assessment of patients with lower urinary tract symptoms and/or benign prostatic hyperplasia. J Urol 1996; 155: 1959-1964.

42. Hsu IC, Lessard E, Weinberg V et al. Comparison of inverse planning simulated annealing and geometrical optimization for prostate high-dose-rate brachytherapy. Brachytherapy 2004; 3: $147-152$.

43. Borghede G, Hedelin H, Holmäng S et al. Combined treatment with temporary short-term high dose rate iridium-192 brachytherapy and external beam radiotherapy for irradiation of localized prostatic carcinoma. Radiother Oncol 1997; 44: 237-244.

44. Barentsz JO, Richenberg J, Clements R et al. ESUR prostate MR guidelines 2012. Eur Radiol 2012; 22: 746-757.

45. Mueller-Lisse UG, Vigneron DB, Hricak $H$ et al. Localized prostate cancer: effect of hormone deprivation therapy measured by using combined three-dimensional ${ }^{1} \mathrm{H}$ MR spectroscopy and MR Imaging: clinicopathologic case-controlled study. Radiology 2001; 221: 380-390.

46. Groenendaal G, van Vulpen M, Pereboom SR et al. The effect of hormonal treatment on conspicuity of prostate cancer: Implications for focal boosting radiotherapy. Radiother Oncol 2012; 103: 233-238.

47. Gabrilove JL, Levine AC, Kirschenbaum A et al. Effect of a GnRH analogue (leuprolide) on benign prostatic hypertrophy. J Clin Endocrinol Metabol 1987; 64: 1331-1333.

48. Murgic J, Chung P, Berlin A et al. Lessons learned using an MRI-only workflow during high-dose-rate brachytherapy for prostate cancer. Brachytherapy 2016; 15: 147-155.

49. Crook J, Ots A, Gaztañaga M et al. Ultrasound-planned highdose-rate prostate brachytherapy: Dose painting to the dominant intraprostatic lesion. Brachytherapy 2014; 13: 433-141. 